\title{
COMPARATIVE STUDY OF ALGORITHMS FOR AUTOMATED GENERALIZATION OF LINEAR OBJECTS
}

\author{
S. Azimjon ${ }^{\text {a }}$, P. K. Gupta ${ }^{\text {b, * }}$, R. S. G. S. Sukhmani ${ }^{\text {b }}$ \\ ${ }^{a}$ Department of Geodesy, Cartography and Cadastre, Faculty of Geography, National University of Uzbekistan - \\ peterazim@gmail.com \\ b Geoinformatics Department, Indian Institute of Remote Sensing - (prasunkgupta, gerapagi)@ gmail.com
}

\section{Commission IV/4}

KEY WORDS: Generalization, Automated road network, Dehradun, CartoSat, Python programming language

\begin{abstract}
:
Automated generalization, rooted from conventional cartography, has become an increasing concern in both geographic information system (GIS) and mapping fields. All geographic phenomenon and the processes are bound to the scale, as it is impossible for human being to observe the Earth and the processes in it without decreasing its scale. To get optimal results, cartographers and map-making agencies develop set of rules and constraints, however these rules are under consideration and topic for many researches up until recent days. Reducing map generating time and giving objectivity is possible by developing automated map generalization algorithms (McMaster and Shea, 1988).
\end{abstract}

Modification of the scale traditionally is a manual process, which requires knowledge of the expert cartographer, and it depends on the experience of the user, which makes the process very subjective as every user may generate different map with same requirements. However, automating generalization based on the cartographic rules and constrains can give consistent result. Also, developing automated system for map generation is the demand of this rapid changing world.

The research that we have conveyed considers only generalization of the roads, as it is one of the indispensable parts of a map. Dehradun city, Uttarakhand state of India was selected as a study area. The study carried out comparative study of the generalization software sets, operations and algorithms available currently, also considers advantages and drawbacks of the existing software used worldwide. Research concludes with the development of road network generalization tool and with the final generalized road map of the study area, which explores the use of open source python programming language and attempts to compare different road network generalization algorithms.

Thus, the paper discusses the alternative solutions for automated generalization of linear objects using GIS-technologies. Research made on automated of road network generalization, which is summarized in this paper, was conducted in IIRS, Dehradun, India.

\section{INTRODUCTION}

\subsection{Background}

All geographic phenomenon and the processes are bound to the scale as it is impossible for human being to observe the Earth and the processes in it without decreasing its scale (Caldwell et $a l, 1984)$. To get optimal results, cartographers and map making agencies develop set of rules and constraints, however these rules are under consideration and topic for many researches up till recent days.

Modification of the scale traditionally is a manual process which requires knowledge of the expert cartographer and it depends on the experience of the user, which makes the process very subjective as every user may generate different map with same requirements (Eckert, 1980). However automating generalization based on the cartographic rules and constrains can give consistent result. Also, developing automated system for map generation is the demand of this rapid changing world (Joao, 1998).
There are three main elements of the map: Road network, Water bodies and the Settlement. These features are essential parts of every map. The research that we have conveyed considered only generalization of the roads, as it is one of the indispensable parts of a map.

Dehradun city, Uttarakhand state of India was selected as a study area (Figure 1), as the city has varies types of roads, like National Highway as well as small minor roads, street width exceeding 18 meters and ones less than 3 meters. Dehradun Guide map scale 1:20000 was taken as an input data and for the assessment of the generalized outcome used Topographic map of Uttaranchal 1:50000, $53 \mathrm{~J} / 3$. The study carried out comparative study of the generalization software set and operation and algorithms used in them, also pointed out advantages and drawbacks of the existing software used worldwide. Research concludes with the development of road network generalization tool and with the final generalized road map of the study area (Sayidov, 2013).

\footnotetext{
* Corresponding author.
} 


\section{AIMS AND OBJECTIVES}

\subsection{Goal}

As aims of the research was taken development of the knowledge based tool for network generalization and this aims composed of several stages that are given in the following lines:

- $\quad$ To prepare road network map of Dehradun city using Dehradun Guide map scale 1:20 000.

- Modifying and correcting digitized map with satellite data: CartoSat-I (PAN Panchromatic) and IRS P6 LISS IV MX (multispectral) merged data.

- Study and assessment of the existing road generalization operators and algorithms

- Developing generalization software for implementing road network generalization and deriving road network map

- $\quad$ Assessing quality of the obtained map.

To accomplish above listed objectives we developed methodology of the research which is represented in Section 2.2 .

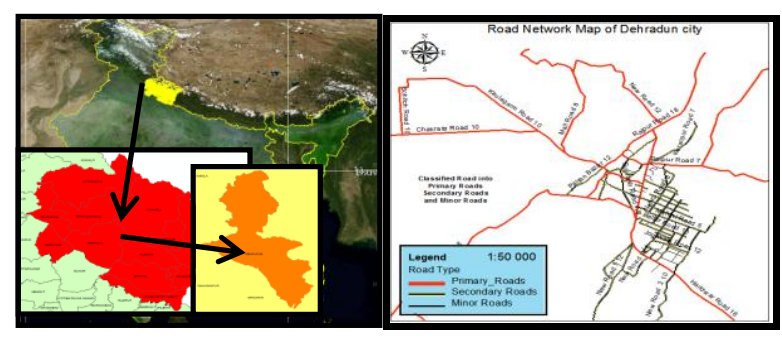

Figure 1: Index Map of the Study Area

\subsection{Methodology}

This project studied algorithms for road networks generalization. First, data collection process is carried out by digitizing a map (1:25 000 Guide Map of Dehradun) and updating it with satellite data (IKONOS). After that, database from digitized data was created, which will serve as an input data for the generalizing software.

Generalizing software was developed by using programming language Python and Graphical User Interface (GUI) of the software is created using PyQt software. This software will have Selection, Elimination, Simplification, and Smoothing operators which is used to generalize the road network. This software studied the trade-offs of time consumption vis-à-vis the accuracy of the implemented algorithms (Figure 2).

Results was assessed on the basis of qualitative and quantitative measures by comparing with Topographic map of part of Uttarakhand (Dehradun) at 1:50 000 scale (Sheet No. $53 \mathrm{~J} / 3$ ).

In order to build a geodatabase for the study, toposheets, Satellite imagery and data collected from field were utilized. The methodology consists of three stages: collection of data, building geodatabase, developing algorithms for road network generalization and applying it to obtain multi-scale maps.

Collection of data also has two parts: generating existing toposheets, maps, Satellite imagery and other data over the study area, and collection of ground truth information to complete the data collection procedure.

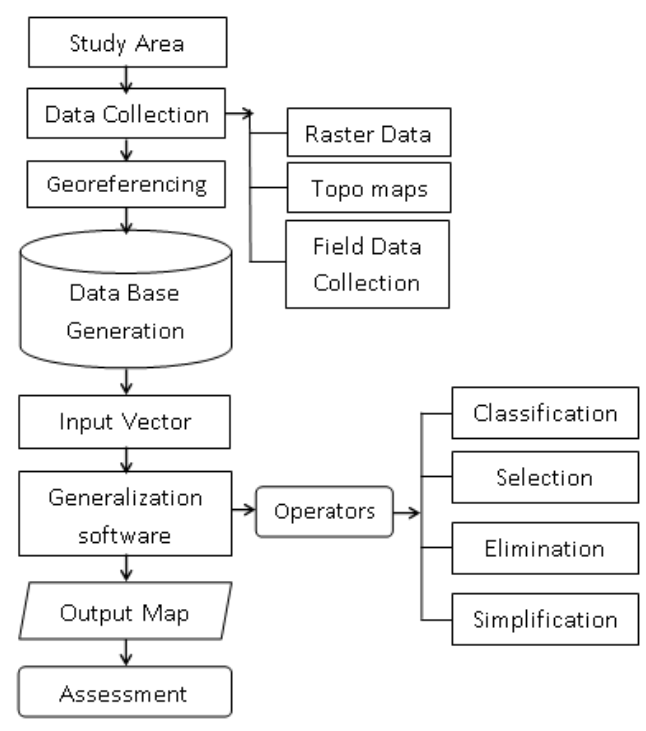

Figure 2: Methodology of the research

To evaluate the result obtained from generalization, "Topographic map of Uttaranchal 1:50 000, $53 \mathrm{~J} / 3$ ", and "Guide map of Dehradun 1:25 000" was used. On-screen digitization and analysis was done using ArcGIS software. Image processing and merging accomplished using ERDAS Imagine software. Algorithms were developed using Python 2.7.3 and GUI was developed using QT Designer 4.8.4 (Figure 3).

Once the data collection process was finished, roads were classified according to their characteristics, which assists the user to generalize the data using developed software. Based on the road classification studies, important characteristics for classification are:

- Width of the roads

- Open areas or,

- Build-up areas

- Plain and rolling terrain

- Mountainous and steep terrain

- Road cover.

The study area that is considered is "Build-up area", mostly plain and covered by asphalt.

\subsubsection{Classification}

A good classification of roads makes the selection easier and more accurate. This step identifies objects that are placed in groups according to similar properties. It also reduces the complexity and will improve the organization of a map. For example, roads were categorized into classes with widths ranging from $3 \mathrm{~m}$ to $18 \mathrm{~m}$, Primary roads, Minor roads and Secondary roads.

\subsubsection{Selection}

Only certain road classes are selected for inclusion at the target scale. Selection is carried out based on the attributes of the roads, like road width, road type (primary, minor or secondary) even in some cases roads maybe selected by their length. 


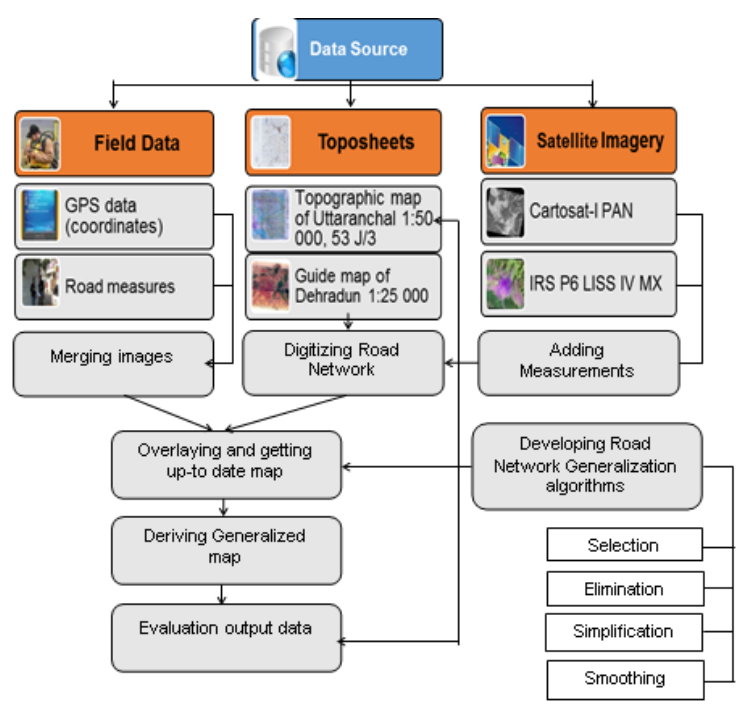

Figure 3: Flowchart of the research

\subsubsection{Elimination}

Mapped features that are not relevant to the map's purpose- e.g. a road branch shorter than a certain length or small road segments that can cause conflict in the final map and are not significant for representation on the map - can be eliminated. In our case, as the target scale is 1:50 000 all the roads having length less than 100 meters should not be represented in the final map. On the other hand, using attribute data of the roads we can further eliminate details obtained at smaller scale maps.

\subsubsection{Simplification}

Based on the research, for line simplification DouglasPeucker's line simplification algorithm is the optimal one. Its positional accuracy is the highest among the other line simplification algorithms and the selected study area is also relatively small so processing time also very unnoticeable (Figure 4).

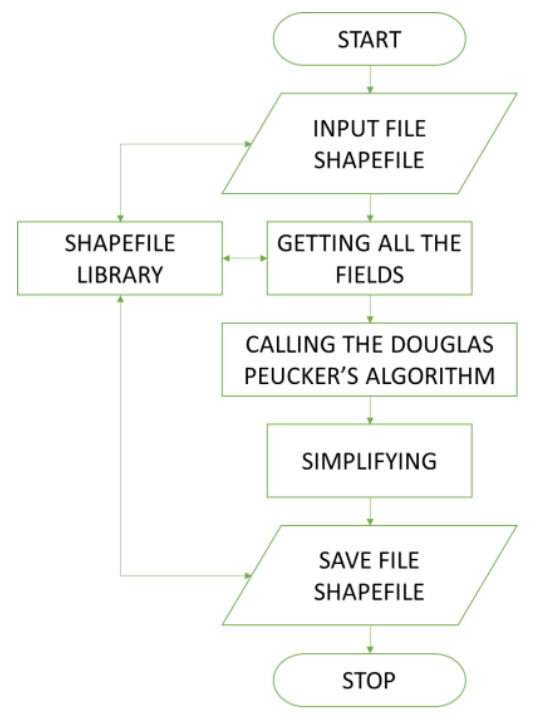

Figure 4: Flowchart of the Douglas Peucker's line simplification operator

\subsubsection{Smoothing}

This operation result in a reduction of plotting time, a reduction of storage space, faster vector-to-raster conversion, and faster vector processing. Line smoothing operation deals with representation of the line with fewer sharp angles to improve aesthetics.

Here, as a smoothing algorithm used Bezier's curve which gives smoothed lines after line simplification algorithm. It gives the line more esthetic view (Figure 5).

Bezier's curve algorithm is widely used in many commercial software, especially it is used in the ArcGIS, ESRI.

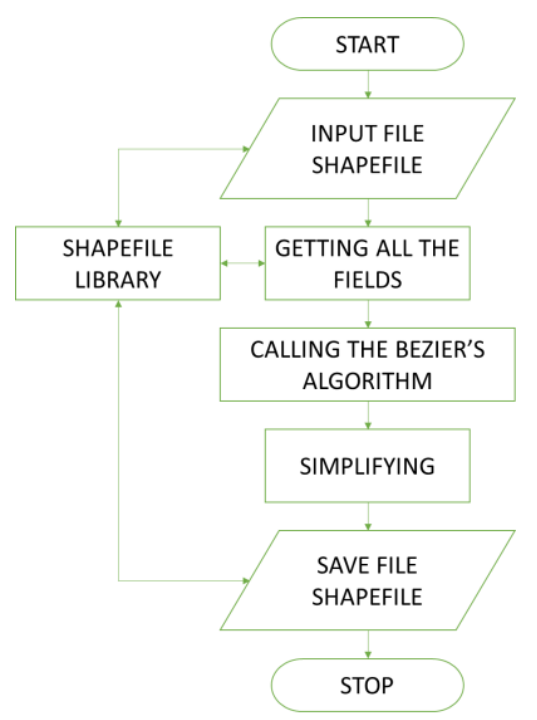

Figure 5: Flowchart of the Beizer's operator

In the Section 3, we present the results obtained from the software and evaluate them.

\section{RESULTS}

As it was mentioned in the previous sections, road generalization tool consists of two operators, "Selection", "Elimination", and two algorithms, "Line Simplification", "Line Smoothing" was developed as a conclusion of the research. Following paragraphs discusses the operators of the software and the accuracy they have.

GUI (Graphical User Interface) was developed in order to accomplish the objectives simultaneously. The GUI has six tabs, "Home", "Selection", "Elimination", "Simplification", "Smoothing" and the "About" (Figure 6).

"Selection" and the "Elimination" operators selects linear features according to the attributes of the roads, such as road class, road width and even by road length. For the 1:50 000 scale roads length less than 200 meters are considered unimportant, which eventually were eliminated.

For simplification of linear features it is believed that the Douglas-Peucker's Line Simplification algorithm gives best results, in positional accuracy as well as in shape distortion. Some researched discuss this algorithm as a time consuming, however in a small data-set as we are using in our research, it is 
accomplishing simplification of the roads within an unnoticeable time.

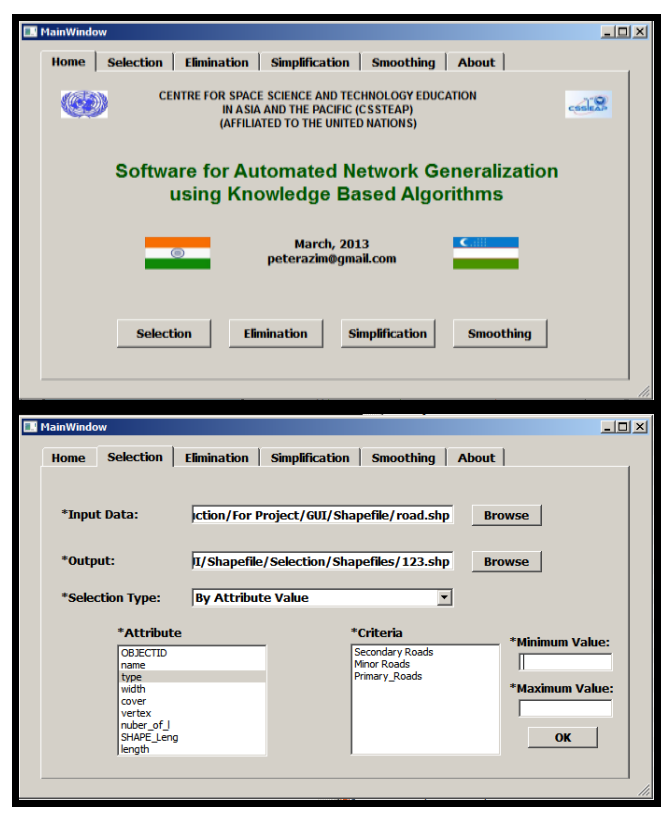

Figure 6: Interface of the generalization tool

The algorithm was experimented with several tolerances and best resembled map with the target map scale 1:50 000 was taken as a road network simplification tolerance for the current map scale.

Overlaying target map and the simplified map results were compared and in most cases the map obtained from the Douglas-Peucker's algorithm were matching with the toposheet, however in some places several dissimilarities were noticed (Figure 7).
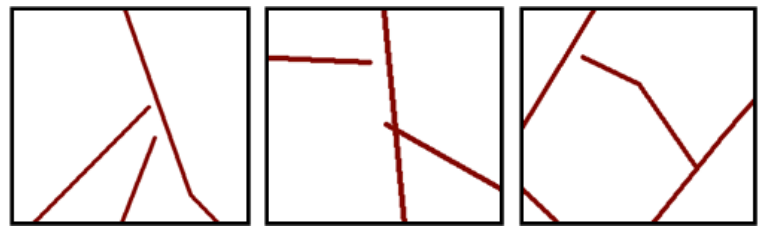

Figure 7: Examples of crossings and errors that was present in the map derived from Douglas-Peucker's Line Simplification algorithm with a tolerance 0.00024 meters

Smoothing operator gives us smoothed lines as after simplification algorithm road looks line straight lines, however in map they need to be smooth and which makes map more readable and enjoyable to use.

Bezier's smoothing algorithm was used and the results were more than sufficient for the given target map, judging from esthetic view and readability of map. However, some positional error was noticed (Figure 8).
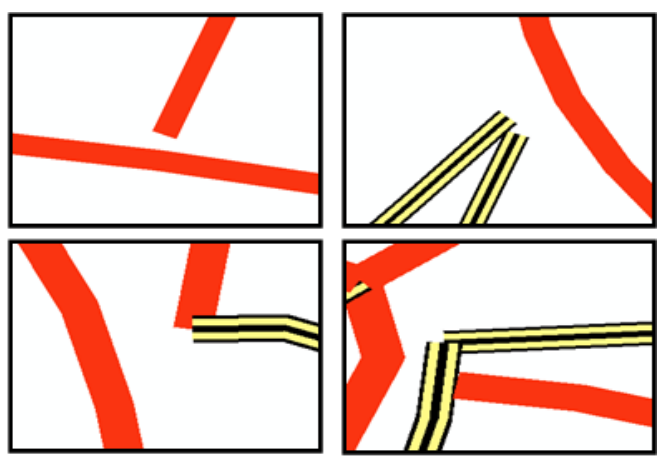

Figure 8: Examples of crossings and errors that was smoothed using Bezier's Algorithm

As final results, digitized road network map and the final generalized output map was compared (Figure 9). It is obvious that, final generalized output map looks more readable compared to the digitized map. Generalized map was also compared to the scanned "Topographic map of Uttaranchal 1:50 $000,53 \mathrm{~J} / 3$ " as the target map was taken as this table (Table 1). Comparison of the roads and differences of them were checked.

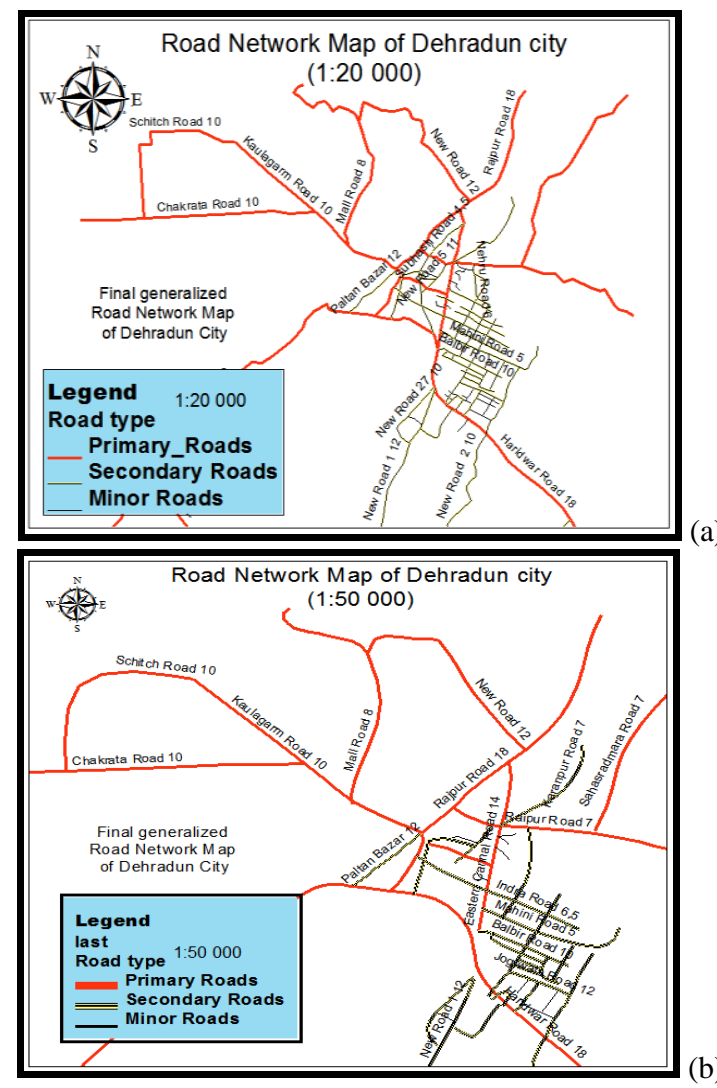

Figure 9: Comparison of the (a) Digitized road network at map scale 1:20 000 and (b) Generalized road network map.

Figure 11 shows that "Primary roads" and the "Secondary roads" are mostly matching, however there are some unnoticeable positional error. Toposheet has 15 "Primary roads" and 23 "Secondary roads", where generalized map 18 and 28 respectively. On the other hand, more differences occurred with "Minor roads", with 5 and 9 respectively. These conditions can be explained with out-datedness of the 
toposheet, as the classification of roads was accomplished based on the field data.

Table 1: Differences of roads in scanned toposheet and generalized output

\begin{tabular}{|l|c|c|c|}
\hline \multicolumn{1}{|c|}{ Roads } & Toposheet & $\begin{array}{c}\text { Output } \\
\text { map }\end{array}$ & Difference \\
\hline $\begin{array}{l}\text { Primary } \\
\text { roads }\end{array}$ & 15 & 18 & 3 \\
\hline $\begin{array}{l}\text { Secondary } \\
\text { roads }\end{array}$ & 23 & 28 & 5 \\
\hline $\begin{array}{l}\text { Minor } \\
\text { roads }\end{array}$ & 5 & 9 & 4 \\
\hline
\end{tabular}

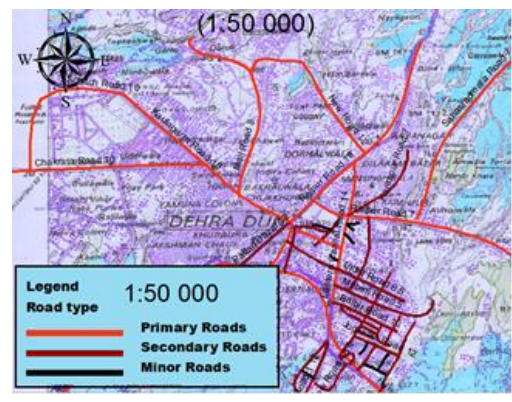

Figure 11: Overlay of the generalized road network map and topographic map of Uttarakhand 1:50 000, $53 \mathrm{~J} / 3$

\section{CONCLUSION}

Main purpose of this study is to study existing automated generalization operator and based on them, develop knowledge based generalization tool of road network and assess obtained results.

Dehradun city, Uttaranchal state, India is selected as a study area. Dehradun Guide map scale 1:20 000 is taken input data and for the assessment generalized data used Topographic map of Uttaranchal 1:50 000,53 J/3. For modification of Guide map CartoSat-I (PAN Panchromatic) and IRS P6 LISS IV MX (multispectral) merged data used.

Literature was reviewed and decided that the existing generalization tools lucking interactive and knowledge based generalization. Also, many line generalization algorithms were observed and concluded that the Douglas-Peucker's line simplification algorithm is the best which gives higher positional accuracy and finished the process in a small area in very unnoticeable time.

Database was created using Guide map of Dehradun and ground truth data were collected and the results were checked by Topographic map of Uttaranchal 1:50 000. Automated road network generalization tool was developed, which has four operators: Selection, Elimination, Simplification and Smoothing. GUI for generalization tool were developed and connected to the operators. Results show that most of the generalized roads, especially Primary roads and Secondary roads match with the toposheet, however there were some more differences in the Minor roads. These conditions can be explained with out-datedness of the toposheet, while classification of roads was accomplished based on the field data, in other words the recent data. The output map was overlaid on the 1:50 000 scale scanned toposheet, which presents some geometric errors. This is due to the simplification and the smoothing parameter values used in these operators. Also, as the Guide map of Dehradun was used to digitize the initial map which is leading to some orthorectification error.

Concluding all of the study, Remote Sensing and Geographic Information Technologies combined with cartographic knowledge can provide knowledge based generalization tool, not only of road network but it could be applied to automated generation of any kind of map, fast as well as accurately. This tool can be used in many map-making organization and can be a big tool for navigation systems, as they require fast map transformation, from different map scales.

\section{ACKNOWLEDGEMENTS}

The authors are grateful to IIRS and CSSTEAP for funding this study. Thanks are due to Director IIRS and CSSTEAP and Head Geoinformatics Department for their technical guidance.

\section{REFERENCES}

Caldwell, D.R., Zoraster, S., and Hugus, M., 1984. Automating Generalization and Displacement Lessons from Manual Methods, Technical Papers of the 44th Annual Meeting of the American Congress on Surveying and Mapping, 11-16 March, Washington, D.C, pp.254-263.

Eckert, Max (1908), "On the Nature of Maps and Map Logic," Trans. by W. Joerg. Bulletin of the American Geographical Society, 40(6):344-351.

Joao, E. M., 1998. Causes and Consequences of Map Generalization. London: Taylor and Francis, pp.25-30.

McMaster, R.B., and K.S. Shea, 1988. Cartographic generalisation in a digital environment: a framework for implementation in a geographic information system. Proceedings of the GIS/LIS'88, 29 November - 30 December 1988, Antonio, Texas, USA, pp.240-249.

Sayidov, A.K., 2013. Comparative study of Knowledge Based Algorithms for Automated Road Network Generalization, (A case study of Dehradun City), Project Report, Indian Institute of Remote Sensing, Dehradun, India. 